\title{
O ENFERMEIRO E A FAMÍLIA dA CRIANÇA COM PERTUBAÇÃO dO ESPETRO dO AUTISMO
}

\author{
Dulce Maria Pereira Garcia Galvão \\ (Pós Doutoramento em Enfermagem, Professora Coordenadora na Escola Superior de Enfermagem de \\ Coimbra - ESEnfC - Coimbra, Portugal.) \\ dgalvao@esenfc.pt \\ Cátia Salomé Simões Marques \\ (Licenciatura em Enfermagem, Escola Superior de Enfermagem de Coimbra - ESEnfC - Coimbra, Portugal.) \\ Dulce Natália Cunha Teixeira \\ (Licenciatura em Enfermagem, Escola Superior de Enfermagem de Coimbra - ESEnfC - Coimbra, Portugal.) \\ Mariana Patrícia Gonçalves Cunha \\ (Licenciatura em Enfermagem, Escola Superior de Enfermagem de Coimbra - ESEnfC - Coimbra, Portugal.) \\ Escola Superior de Enfermagem de Coimbra. Avenida Bissaya Barreto, apartado 55. CEP 3001-901. Coimbra, \\ Portugal.
}

\begin{abstract}
RESUMO
Antecedentes: As Perturbações do Espetro do Autismo (PEA), perturbações globais do desenvolvimento, têm incidência de 4-5 por cada 10000 nascimentos, predominantes no sexo masculino (razão de 4:1), apresentam-se em três níveis de gravidade que interferem na vida da criança, com impacto na dinâmica e funcionamento familiar. Objetivos: Conhecer como se desenvolve a consulta de enfermagem junto da família da criança com PEA, identificar o que avaliam e que planos de cuidados os enfermeiros desenvolvem quando assistem a família da criança; compreender as dificuldades dos enfermeiros na consulta de enfermagem e a relação que estabelecem com a família da criança. Participantes e Métodos: Revisão Integrativa da Literatura, para responder à questão "A que aspetos estão atentos os enfermeiros quando assistem a família da criança com PEA na consulta de enfermagem?". Pesquisa bibliográfica no portal EBSCO, B-On acedendo às bases de dados Psychology and Behavioral Sciences Collection; MEDLINE; CINAHL Plus; MedicLatina, ERIC, Science Citation Index Expanded, SciVerse ScienceDirect e PMC, base de dados SCielo e Google Académico, selecionando estudos de 2009- Maio/2015 em português, inglês e espanhol. Utilizaramse os descritores "nurse"; "nursing"; "family"; "autism"; "autismo spectrum disorder"; "knowledge" e correspondentes em português. Incluíram-se sete artigos. Resultados: Os enfermeiros centram a atuação na criança, realizam avaliação do desenvolvimento infantil e conhecimento da família sobre
\end{abstract}




\section{O ENFERMEIRO E A FAMÍLIA DA CRIANÇA COM PERTUBAÇÃO DO ESPETRO dO AUTISMO}

a perturbação, identificando necessidades da família. 0 plano de cuidados é realizado com foco na criança relativamente às rotinas e atividades ritualizadas, sem especificidade para a família. As dificuldades dos enfermeiros centram-se na falta de conhecimento sobre a perturbação e abordagem à família. A relação do enfermeiro e família baseia-se na confiança, compreensão e aceitação. 0 enfermeiro adota papel socializador e educador. Conclusões: Os enfermeiros não se sentem preparados para lidar com a família da criança, necessitam formação. Têm de direcionar a prática para as necessidades dos pais e irmãos.

Palavras-chave: transtorno autístico; família; papel do profissional de enfermagem

\section{ABSTRACT}

The nurse and the family of the child with Autism Spectrum Disorder.

Introduction: The Autism Spectrum Disorders (ASD), global disturbances of child development, has an incidence of 4-5 per 10,000 births, prevalence in males (ratio of 4: 1), presents in three levels of severity that interfere in the life of the child, with impact on family dynamics and operation. Objectives: Knowing how the nursing appointment with the family of the child with ASD develops, identifying what the nurses assess and which care plans they develop when they assist the child's family; understanding the difficulties of the nurses in the nursing appointment as well as the relationship they establish with the child's family. Methods: Integrative Literature Review, to answer the question "At which aspects are the nurses attentive in the nursing appointment when they assist the family of the child with ASD?". Bibliographic search in databases Psychology and Behavioral Sciences Collection, MEDLINE, CINAHL Plus, MedicLatina, ERIC, Science Citation Index Expanded, SciVerse ScienceDirect, PMC, SCielo and Google Académico, selecting studies from 2009 to May/2015 in Portuguese, English and Spanish, using the descriptors "nurse"; "nursing"; "family"; "autism"; "autism spectrum disorder"; "knowledge" and the corresponding words in Portuguese. Seven articles were included. Results: The Nurses focus their actuation on the child, they assess the child development and the family knowledge about the disorder, identifying the family needs. The care plan is focused on the child, specifically, in which concerns to the routines and ritualized activities, without family specificities. The difficulties of the nurses are related to the lack of knowledge about the disorder and to the approach to the family. The relationship of the nurse with the family is based on trust, understanding and acceptance. The nurse plays a role of socializer and educator. Conclusion: The Nurses do not feel prepared to deal with the family, requiring training. They must direct their practices to the needs of parents and brothers.

Key-words: autistic disorder; family; nurse's role

\section{INTRODUÇÃO}

As Perturbações do Espetro do Autismo (PEA), perturbações globais do desenvolvimento, predominantes no sexo masculino com uma razão de 4:1, não são raras. A sua incidência é de 4-5 por cada 10000 nascimentos (Zanatta, Menegazzo, Guimarães, Ferraz e Motta, 2014; Ordem dos Enfermeiros, 2014; Pereira, 2005 citado por Gonçalves, 2011). A Associação Psiquiátrica Americana (APA) (2013, p.31) define-as como “(...) um síndroma neuro-comportamental com origem em perturbações do sistema nervoso central que afectam o normal desenvolvimento da criança. Os sintomas ocorrem nos primeiros três anos de vida e incluem três grandes domínios de perturbação: social, comportamental e comunicacional." Refere existirem três níveis de gravidade, sendo o mais grave 0 de nível três.

As características das crianças com PEA variam em função do nível de desenvolvimento e idade cronológica (Gonçalves, 2011). A existência de sinais de PEA na criança são precoces (Ordem dos Enfermeiros, 2014). A manifestação do autismo desencadeia mudanças em toda a dinâmica fami- 
liar, em especial na relação mãe-filho (Nunes e Santos, 2010 citado por Zanatta et al. 2014). A dinâmica familiar também sofre alterações a nível financeiro, qualidade de vida física e psíquica bem como social dos membros (Fávero e Santos, 2005). A PEA é uma fonte de stress na família e quando acompanhada de prejuízo cognitivo torna-se o fator major de stress parental (Fávero e Santos, 2005).

Além de cuidar a criança os profissionais de enfermagem também assistem a família, sendo esta considerada como uma unidade e reconhecida como uma constante na vida da criança. 0 ajustamento à nova situação deve ser vigiado pelos enfermeiros (Althoff, Renck e Sakae, 2005). Cuidar da família é um compromisso do enfermeiro.

Neste contexto, questionámo-nos "A que aspetos estão atentos os enfermeiros quando assistem a família da criança com PEA na consulta de enfermagem?". Estabelecemos como objetivo geral: Conhecer como se desenvolve a consulta de enfermagem junto da família da criança com PEA, e como objetivos específicos: Identificar o que avaliam os enfermeiros quando assistem a família da criança com PEA; Identificar os planos de cuidados que os enfermeiros desenvolvem quando assistem a família da criança com PEA; Compreender as dificuldades que os enfermeiros manifestam quando lidam com a família da criança com PEA; Identificar a relação que os enfermeiros estabelecem com a família da criança com PEA.

\section{MÉTODOS}

Revisão integrativa da literatura utilizando-se as palavras: "nurse", "nursing", "family", "autism", "autismo spectrum disorder", "knowledge". Estabelecemos como critérios de inclusão que os estudos incluíssem, independente da metodologia de investigação utilizada, as famílias de crianças com PEA assistidas em consultas de enfermagem. Tivéssemos acesso gratuito ao texto integral, que os estudos fossem publicados entre 2009 e maio de 2015, e escritos em Português, Inglês ou Espanhol. Excluímos estudos que incluíssem famílias de crianças com PEA com outra perturbação/situação patológica e estudos não científicos. Consultámos o Portal EBSCO e as bases de dados: Psychology and Behavioral Sciences Collection; Medline; CINAHL; MedicLatina, ERIC. Identificaram-se 217 artigos, seleccionaram-se três e utilizou-se um. Consultámos também o Portal B-on e as bases de dados: MEDLINE/PubMed, Science Citation Index Expanded, SciVerse ScienceDirect, PMC. Identificaram-se 27 artigos, seleccionaram-se três e utilizou-se um. Consultámos também a Scielo de onde utilizámos um artigo. Fizemos ainda busca no Google Académico de onde utilizámos quatro artigos.

\section{RESULTADOS}

Do total dos sete artigos, quatro faziam referência ao que os enfermeiros avaliam quando assistem a família da criança com PEA; quatro abordavam o que os enfermeiros incluíam nos planos de cuidados de enfermagem na consulta de enfermagem à família da criança com PEA, seis incluíam as dificuldades dos enfermeiros quando lidam com a família de crianças com PEA e dois faziam referência à relação que os enfermeiros estabeleciam com a família da criança com PEA.

\section{Avaliação dos enfermeiros quando assistem a família da criança com PEA}

Nunes, Souza e Giunco (2009) no estudo que desenvolveram sobre conhecimento da equipa de enfermagem e autismo, junto de 78 enfermeiros, apuraram que os enfermeiros ao avaliarem a família verificaram que esta não conhece a idade de início da manifestação da doença da criança, nem identificam corretamente as alterações que a criança apresenta o que leva ao prejuízo do diagnóstico e tratamento por procurarem ajuda tardiamente. Verificaram também que os enfermeiros fazem a avaliação do desenvolvimento infantil da criança. 


\section{O ENFERMEIRO E A FAMÍLIA DA CRIANÇA COM PERTUBAÇÃO DO ESPETRO DO AUTISMO}

Carniel, Saldanha e Fensterseifer (2010) desenvolveram um estudo para analisar a atuação do enfermeiro face à criança com PEA, com uma amostra de cinco enfermeiros. Constataram que 0 enfermeiro identifica as metas e necessidades da família e planeia intervenções que vão de encontro aos problemas definidos.

Nogueira e Martins do Rio, em 2011, para identificar o impacto que a PEA traz à família recorreram à mãe de um adolescente com 15 anos portador de PEA. Apuraram que os enfermeiros durante a entrevista abordaram necessidades a nível de apoios governamentais, apoio dos profissionais de saúde e educação para as crianças.

Para conhecer a perceção da equipa de enfermagem face ao atendimento de crianças com PEA Dartora, Mendieta e Franchini (2014), desenvolveram um estudo com seis enfermeiros. 0 estudo mostrou que os enfermeiros referem que é necessário um olhar mais atento durante a avaliação para compreender 0 que a criança com PEA está a expressar. Os enfermeiros efetuam a recolha de dados para definirem diagnósticos e intervenções adequadas à criança com PEA.

Plano de cuidados de enfermagem para a consulta de enfermagem à família da criança com PEA

Nunes, Souza e Giunco (2009) no estudo que realizaram sobre o conhecimento da equipa de enfermagem e PEA verificaram que os enfermeiros reconhecem o isolamento social como um sintoma clássico da PEA e identificam os sintomas presentes na criança de não manter contacto visual e hipoatividade mas não reconhecem as alterações relatadas pelos pais: "não chorar" e "não gosta de colo". Para os enfermeiros as crianças com PEA apresentam alterações de preferência alimentar, o que conduz a estados de sobrenutrição ou desnutrição.

No estudo de Carniel, Saldanha e Fensterseifer (2010), observou-se que os enfermeiros devem ajudar os pais a aliviar a culpa e vergonha muitas vezes associada à PEA. Como intervenções referem explicar o que é a PEA e incentivar os pais a encontrar mais informações sobre a PEA.

Também Carniel, Saldanha e Fensterseifer, desenvolveram, em 2011, o estudo "Proposta de um plano de cuidados para crianças autistas" junto de cinco enfermeiros para propor um plano de cuidados à criança com PEA e sua família. Apuraram que foram elaborados 6 diagnósticos de enfermagem: Risco de automutilação; Interação social prejudicada; Comunicação verbal prejudicada; Distúrbio da identidade pessoal; Risco de desenvolvimento retardado e Risco de stress por mudanças.

Nogueira e Martins do Rio (2011) verificaram que face ao diagnóstico, a família fica em estado de "choque", entra numa fase de negação, sendo que a aceitação é gradual e nunca se conclui. A família expressa sentimentos de ansiedade e preocupações, relacionadas com o que poderão fazer, e revolta pela forma como foram tratados, por não existirem apoios e por verificar que a sociedade não se preocupa, falta de informação sobre a doença, problemas de desenvolvimento que a criança sofre, educação da criança, recursos económicos, saúde e o bem-estar dos pais, relações familiares, relação conjugal, relações sociais foram dificuldades e alterações manifestadas na família.

\section{Dificuldades dos enfermeiros quando lidam com a família da criança com PEA}

No estudo de Nunes, Souza e Giunco (2009) os enfermeiros afirmaram possuir pouco conhecimento sobre 0 tema. Maior dificuldade do enfermeiro prende-se com esclarecimentos que têm de dar devido às necessidades exigidas pela PEA. Reconheceram que as mães são os membros da família com maior prejuízo tanto emocional, profissional como conjugal. Os enfermeiros além de não se sentirem capazes de abordar corretamente a família, também apresentaram desinteresse quanto ao diagnóstico da perturbação. Os enfermeiros reconhecem a importância da avaliação por uma equipa multiprofissional. 
Carniel, Saldanha e Fensterseifer (2010) verificaram que os enfermeiros relataram que é necessário um estudo mais aprofundado sobre PEA.

Hall e Graff em 2010 desenvolveram um estudo para investigar como os pais visualizam os comportamentos dos filhos com PEA, junto de pais de crianças com PEA inseridos num grupo de suporte (nove pais: sete mães e dois pais); duas avós (cuidadoras primárias) e verificaram que embora os pais considerassem que os profissionais de saúde fossem uma importante fonte de suporte e de recursos possuíam menos conhecimento sobre a PEA do que eles próprios.

No estudo de Nogueira e Martins do Rio (2011), relativamente às atitudes dos profissionais de saúde, a mãe mencionou como maiores dificuldades o facto dos profissionais de saúde não valorizarem as queixas e encararem as opiniões dos pais como irrelevantes.

Em 2013, Will, Barnfather e Lesley, com o objetivo de examinar como os enfermeiros dos cuidados de saúde primários percecionam o nível de competência a prestar cuidados a crianças com PEA elaboraram um estudo com trinta e sete enfermeiros. Verificaram que os enfermeiros se sentem menos competentes a prestarem cuidados a crianças com PEA do que a crianças com outra doença crónica. 0 estudo mostrou ainda que as barreiras percebidas pelos enfermeiros nos cuidados à criança com PEA são maiores do que nas crianças com outra patologia crónica e passam por: falta de coordenação de cuidados, família ser reticente em relação às vacinas, falta de tempo durante a consulta, falta de conhecimento sobre PEA, falta de directrizes para a prática, falta de reembolsos, frequência de medicinas alternativas complementares, utentes que utilizam como recursos outros profissionais e família reticente em relação à medicina tradicional.

Dartora, Mendieta e Franchini (2014) verificaram que os enfermeiros apresentam uma "ideia pré-formada" sobre as crianças com PEA associada a alguém diferente e constataram que revelaram não ter adquirido um ensino adequado sobre PEA.

\section{Relação dos enfermeiros com a família}

Carniel, Saldanha e Fensterseifer (2010) e Dartora, Mendieta e Franchini (2014) nos estudos que desenvolveram reconheceram que 0 enfermeiro assume um papel de socializador junto da criança com PEA com o objetivo de inclusão da criança na sociedade. Com a família 0 enfermeiro apresenta um papel de educador. Transmite-Ihe informações acerca da PEA com base numa relação de confiança, aceitação, apoio e compreensão.

\section{DISCUSSÃO}

A categoria "Avaliação dos enfermeiros quando assistem a família da criança com PEA" permitiu-nos ver que nos estudos de Nunes, Souza e Giunco (2009), Nogueira e Martins do Rio (2011) e Dartora, Mendieta e Franchini (2014) os enfermeiros centraram a sua atuação mais na criança. Porém, na consulta de enfermagem, intervenção realizada pelo enfermeiro com vista a realizar uma avaliação, ou planeamento de cuidados de enfermagem, no sentido de ajudar 0 indivíduo a atingir a máxima capacidade de autocuidado (Portaria № 306-A de 20 de Dezembro de 2011), os enfermeiros lidam com a criança com PEA e sua família. As intervenções que os enfermeiros desenvolvem no decorrer das consultas de enfermagem de vigilância de Saúde Infantil têm em vista a obtenção contínua de ganhos em saúde (Ministério da Saúde, 2013). Nestas consultas, os enfermeiros desenvolvem ações junto da criança e sua família que Ihes permitem: Sinalizar e proporcionar apoio continuado à criança com doença crónica/deficiência e à sua família (Ministério da Saúde,2013). Cabe aos enfermeiros identificar, numa perspetiva centrada na família, as necessidades especiais de cada criança e sua família, sinalizá-las, proporcionar-lhes apoio continuado e promover a articulação entre os intervenientes nos cuidados (Pinto et al., 2010). Para Pinto et al. (2010) o enfermeiro deve 


\section{O ENFERMEIRO E A FAMÍLIA DA CRIANÇA COM PERTUBAÇÃO DO ESPETRO dO AUTISMO}

promover o relacionamento com a família, de forma a prestar cuidados que permitam o enfrentamento de dificuldades pela família, especialmente em situação de doença. Acrescentam que na prestação de cuidados de enfermagem todos os membros são reconhecidos como o foco de cuidado e não somente a criança mas também as necessidades reveladas pela família (Pinto et al., 2010). Nos resultados obtidos por Carniel, Saldanha e Fensterseifer (2010) observou-se que os enfermeiros devem identificar as metas e necessidades da família, sendo este dado compartilhado com Pinto et al. (2010) em que afirmam que cabe aos enfermeiros identificar, numa perspetiva centrada na família, as necessidades especiais de cada criança e sua família.

Na categoria "Plano de cuidados de enfermagem para a consulta de enfermagem à família da criança com PEA" verifica-se que o foco de atenção do plano de cuidados de enfermagem dos estudos de Nunes, Souza e Giunco (2009) e Carniel, Saldanha e Fensterseifer (2011) é a criança. Os diagnósticos de enfermagem elaborados e apresentados têm como base os sinais e sintomas manifestados pela criança com PEA, atendendo às alterações relacionadas com o comportamento, comunicação e interação social.

A criança com PEA sente, olha e percebe o mundo de maneira diferente, e cabe ao enfermeiro entender a singularidade de cada criança. Os enfermeiros devem percecionar a inflexibilidade para as mudanças de rotinas e atividades ritualizadas (Dartora, Mendieta e Franchini, 2014). 0 enfermeiro deve considerar a estimulação da comunicação bem como 0 desenvolvimento das relações com outros indivíduos e consequentes alterações de rotina (Zanatta et al., 2014). Tal como verificado por Gonçalves (2011) o tratamento da PEA é adaptado ao desenvolvimento da criança, onde a prioridade insere nas áreas com alterações, terapia da fala, interação social e educação/suporte familiar. Zanatta et al. (2014) acrescentam ser necessário que o profissional de enfermagem possua conhecimentos sobre autismo, de forma a estabelecer um plano de cuidados para a família para desempenhar os cuidados adequados.

Nogueira e Martins do Rio (2011) identificaram que a família experiencia sentimentos de negação, com gradual aceitação, com presença contínua de ansiedade, e preocupações quanto aos apoios governamentais, de saúde e sociais. Estas alterações manifestadas pela família, relacionadas com a dificuldade em aceitar o diagnóstico e a necessidade de cuidados especiais, conduz a prejuízo emocional em todos os membros da família. Tanto Fávero e Santos (2005) como Monteiro et al. (2008) ressalvam o impacto na dinâmica familiar a nível financeiro, qualidade de vida física e psíquica bem como social dos membros. Estas alterações têm especial impacto na relação mãe-filho (Nunes e Santos, 2010 citado por Zanatta et al., 2014).

Quanto à categoria "Dificuldades dos enfermeiros quando lidam com a família da criança com PEA" a falta de conhecimento é a dificuldade mais expressa pelos profissionais ao lidar com a criança. Apesar das PEA não serem raras, com uma incidência de 4-5 crianças por cada 10000 nascimentos verifica-se através dos resultados que 0 conhecimento para a abordagem das mesmas não está solidificado, afetando a prestação de cuidados pelo enfermeiro (Zanatta et al., 2014; Dartora, Mendieta e Franchini, 2014; Ordem dos Enfermeiros, 2014; Gonçalves, 2011). Todavia, segundo Zanatta et al. (2014) os enfermeiros possuem o papel de educadores, ou seja, ao prestar cuidados à família devem ter a competência de informar sobre a PEA, as redes de apoio e estratégias de desenvolvimento da criança. Também, Althoff, Renck e Sakae (2005) afirmam que é função do enfermeiro a capacitação da família para a prestação de cuidados bem como elucidação sobre a situação.

Quanto à "Relação dos enfermeiros com a família" apresenta-se dividida, existindo as relações estabelecidas com a família e com a criança. De acordo com Carniel, Saldanha e Fensterseifer (2010) o papel do enfermeiro é a inclusão da criança na sociedade. As características da PEA, como 
por exemplo, os movimentos estereotipados e a incapacidade de criar uma relação como referem Martins (2012) e Coelho e Santo (2006), o enfermeiro deve construir em conjunto com a criança habilidades sociais. Como verificado por Moreira (2010) as crianças com PEA não têm medo de perigos, e como tal é necessário protegê-la de eventuais acidentes, em que 0 enfermeiro deve transmitir estas informações à família baseando-se numa relação de confiança. Carniel, Saldanha e Fensterseifer (2010) refere ainda que juntamente com a família o enfermeiro deve estabelecer limites ao comportamento da criança, baseado numa relação de aceitação e compreensão. Baseado numa relação de confiança, o cuidado à família exercido pelo enfermeiro no papel de educador consiste em informar sobre 0 autismo, sobre as redes de apoio centrando-se nas estratégias para ajudar no desenvolvimento da criança (Zanatta et al., 2014).

Também Dartora, Mendieta e Franchini (2014) referem que o enfermeiro deve assumir um papel, socializador, de aceitação e compreensão para estabelecer limites, orientação e apoio à família. Zanatta et al (2014) bem como Fávero e Santos (2005) verificaram que as estereotipias e 0 isolamento da criança são fonte de isolamento social da família, esta vivencia sentimentos de culpa e vergonha associados à severidade da manifestação dos sintomas bem como a agressividade. Assim, na abordagem à família é fundamental a compreensão e aceitação da doença, e incentivar os pais na participação no tratamento (Carniel, Saldanha e Fensterseifer, 2010). 0 enfermeiro exerce um papel de agente socializador que tem como objetivo promover a inclusão da criança autista na sociedade (Zanatta et al., 2014).

\section{CONCLUSÃO}

Pretendeu-se conhecer como se desenvolve a consulta de enfermagem junto da família da criança com PEA. Os estudos mostraram que não existem cuidados de enfermagem direcionados à família da criança com PEA. Os enfermeiros possuem pouco conhecimento sobre PEA apesar da sua prevalência. Apenas o estudo de Carniel, Saldanha e Fensterseifer (2010) demonstrou que na avaliação os enfermeiros avaliam as metas e necessidades da família, porém nenhum tem uma visão global e sistémica da família. Os enfermeiros centram a atuação na criança, realizam avaliação do desenvolvimento infantil e conhecimento da família sobre a perturbação, identificando como sendo estas as necessidades da família. 0 plano de cuidados é realizado com foco na criança relativamente às rotinas e atividades ritualizadas, sem especificidade para a família. Somente 0 estudo de Nogueira e Martins do Rio (2011) a par dos problemas centrados na criança revela que a família expressa revolta por verificar que a sociedade não se preocupa com a saúde e o bem-estar dos pais, relações familiares, relação conjugal e relações sociais. As dificuldades dos enfermeiros centram-se na falta de conhecimento sobre a perturbação e abordagem à família. A relação do enfermeiro e família baseia-se na confiança, compreensão e aceitação. 0 enfermeiro adota papel socializador e educador. Os enfermeiros não se sentem preparados para lidar com a família da criança com PEA. Têm de direcionar a prática para as necessidades dos pais e irmãos. Consideramos que uma das estratégias a implementar passa, primeiramente, pela formação dos profissionais que exercem a sua prática no âmbito da Saúde Infantil. Assim os enfermeiros poderão assistir e ensinar a família, dando resposta às necessidades da família da criança com PEA.

\section{REFERÊNCIAS BIBLIOGRÁFICAS}

Althoff, C., Renck, L., Sakae S. (2005). Famílias de criança que necessitam de cuidados especiais: 0 impacto sobre a vida familiar. Família, saúde e desenvolvimento: Curitiba, 7(3), 221-229.

APA. (2013). DSM 5: Diagnostic and statistical manual of mental disorders (5th ed.). England. American Psychiatric Publishing. 


\section{O ENFERMEIRO E A FAMÍLIA DA CRIANÇA COM PERTUBAÇÃO DO ESPETRO DO AUTISMO}

Carniel, E. L., Saldanha, L. B., Fensterseifer, L. M. (2010). A atuação do enfermeiro frente à criança autista. Pediatria (São Paulo), 32(4), 255-260.

Carniel, E. L., Saldanha, L. B., Fensterseifer, L. M. (2011). Proposta de um plano de cuidados para crianças autistas. Pediatria (São Paulo), 33(1), 4-8.

Coelho, M., Santo, A. (2006). Autismo: Perda de contacto com a realidade exterior. (Acção de Formação nº 07/2006) Centro de Formação Contínua de Professores de Ourique, Castro Verde, Aljustrel e Almodôvar, Portugal.

Dartora, D. D., Mendieta, M. C., Franchini, B. (2014). A equipa de enfermagem e as crianças autistas. Journal of Nursing and Health, 4(1), 27-38.

Fávero, M. A. B., Santos M. A. (2005). Autismo Infantil e estresse familiar: uma revisão sistemática da literatura. Psicologia: Reflexão e Crítica, 18(3), 358-369.

Gonçalves, A. (2011). Os modelos de intervenção são eficazes para melhorar a inclusão de crianças com autismo (Dissertação de Mestrado). Escola Superior de Educação Almeida Garret, Portugal.

Hall, H., Graff, J. C. (2010) Parenting Challenges in families of children with autism: a pilot study. Issues in Compreensive Pediatric Nursing, 33, 187-204.

Martins, C. P. (2012). Face a face com o autismo: será a inclusão um mito ou uma realidade? (Dissertação de Mestrado). Escola Superior de Educação João de Deus, Portugal.

Ministério da Saúde. (2013). Programa Nacional de Saúde Infantil e Juvenil. DGS. Monteiro, C. F., Batista, D. 0., Moraes, E. G., Magalhães, T. S., Nunes, B. M., Moura, M.

E. (2008). Vivências maternas na realidade de ter um filho autista: uma compreensão pela enfermagem. Revista Brasileira de Enfermagem, 61(3), 330-350.

Moreira, N. (2010). 0 cuidar do portador de autismo e seus familiares: uma abordagem mutiprofissional. Revista de pesquisa: cuidado é fundamental online, 2, $271-274$.

Nogueira, M. A. A., Martins do Rio, S. C. M. (2011). A família com criança autista: Apoio de Enfermagem. Revista Portuguesa de Enfermagem de Saúde Mental, 5, 16-20.

Nunes, S. C., Souza, T. Z., Giunco, C. T. (2009). Autismo: Conhecimento da equipe de enfermagem. CuidArte Enfermagem, 3(2), 134-141.

Ordem dos Enfermeiros (2014). A criança com autismo. Jornal da Madeira.Recuperado de http://www.ordemenfermeiros.pt/sites/madeira/informacao/Paginas/artigos2014.aspx Pinto, J.

P., Ribeiro, C. A., Pettengill, M. M., Balieiro, M., M. (2010). Cuidado centrado na

família e sua aplicação na enfermagem pediátrica. Revista Brasileira de Enfermagem, 63(1),132-135.

Portaria no 306-A/2011. Diário da República no 242 de 20 de Dezembro de 2011, I Série.

Ministério das Finanças e da Saúde. Lisboa, Portugal.

Will, D., Barnfather, J., Lesley, M. (2013). Self-Perceived Autism Competency of Primary Care Nurse Practitioners. The Journal for Nurses Practitioners, 9(6), 350-351.

Zanatta, E. A., Menegazzo E., Guimaraes, A. E., Ferraz L., Motta, M. G. C. (2014). Cotidiano de famílias que convivem com 0 autismo infantil. Revista Baiana de Enfermagem, 28(3), 271-282. 